\title{
Impact Des Eaux Usées Et Des Aménagements Urbains Sur La Répartition Des Espèces De Culicidae (Diptera Nematocera) Dans La Ville De Meknès (Maroc)
}

\author{
Dr. Handaq Nadia \\ Pr. Blenzar Abdelali \\ University Moulay Ismail, Faculty of Sciences, Zitoune, Meknès, Morocco \\ doi: 10.19044/esj.2017.v13n27p184 URL:http://dx.doi.org/10.19044/esj.2017.v13n27p184
}

\begin{abstract}
In the Meknes region, urban and peri-urban green spaces are mainly irrigated by wastewater, whose flow has considerably increased in recent years due to the extent of urbanization. These agricultural areas along the three valleys of Bou Ishak, Boufekrane and Ouislane are an asset of the city of Meknes, but they are at the origin of the proliferation of culicidal species harmful to the urban population, especially during the summer season marked by the decrease in clear water from sources. The biotypological analysis of the breeding sites encountered in the studied perimeter, revealed the main elements that determine the distribution of culicidal species. This depends in large part on the situation of the deposits, either upstream of the discharges of the waste water or downstream of these discharges. Thus, deposits located in the southern suburbs of Meknes, upstream of domestic and industrial wastewater discharges, show an important specific richness with the presence of eleven species divided into four genera. On the other hand, in the lodgings located in the urban perimeter, characterized by polluted water, we noted the predominance of two species belonging to two genera Culex and Aedes. The reduction in the specific richness of these deposits is related to the degradation of water quality, mainly due to the discharge of domestic and industrial wastewater.
\end{abstract}

Keywords : Wastewater, Larval breeding sites, Culicids, Meknes, Morocco

Résumé

Dans la région de Meknès, les espaces verts urbains et péri-urbains sont principalement irrigués par les eaux usées, dont le débit a considérablement augmenté, ces dernières années, par l'ampleur de 
l'urbanisation. Ces espaces agricoles longeant les trois vallées de Bou Ishak, Boufekrane et Ouislane constituent un atout de la ville de Meknès, cependant ils sont à l'origine de la prolifération des espèces culicidiennes nuisibles à la population urbaine, surtout pendant la saison estivale marquée par la diminution des eaux claires des sources. L'analyse et le suivi des gîtes larvaires explorés dans le périmètre étudié, a permis de mettre en évidence les principaux éléments qui conditionnent la répartition des espèces culicidiennes. Celle-ci dépend en grande partie de la situation des gîtes, soit en amont des rejets des eaux usées, soit à l'aval de ces rejets. Ainsi, les gîtes situés dans la banlieue sud de Meknès, en amont des rejets des eaux usées domestiques et industrielles, montrent une richesse spécifique importante avec la présence de onze espèces réparties en quatre genres. Par contre dans les gîtes situés dans le périmètre urbain, caractérisés par des eaux polluées, nous avons noté la prédominance de deux espèces appartenant à deux genres Culex et Aedes. La réduction de la richesse spécifique constatée au niveau de ces gîtes, est en relation avec la dégradation de la qualité des eaux, liée principalement aux rejets des eaux usées domestiques et industrielles.

Mots-clés: Eaux usées, Gîtes larvaires, Culicidae, Richesse spécifique, Meknès, Maroc

\section{Introduction}

Parmi les insectes hématophages piqueurs suceurs, les Culicidae (Diptera Nematocera), sont ceux qui ont fait l'objet de nombreux travaux de recherche. Si les Anophèles, vecteurs du paludisme, ont été les plus étudiés au Maroc vu leur rôle vectoriel (Guy, 1963 ; Guy et Holsteum, 1968 ; Guy et al., 1976 ; Bailly Choumara, 1965, 1967, 1973 a et b; Metge, 1986, 1991 ; Bouallam, 1992 ; Alaoui Slimani et al., 1999 ; Faraj et al., 2006, 2008), les travaux sur les autres genres tels que Aedes et Culex restent relativement limités. La lutte contre les maladies transmises par la piqûre de ces moustiques d'une part, et la lutte contre la lourde nuisance qu'elles causent, d'autre part, font de ces Culicides un matériel d'étude important pour les entomologistes.

Les premiers travaux se rapportant aux Culicidae du Maroc sont particulièrement importants (Seguy, 1930 ; Messerlin, 1938; Roubaud, 1935 ; Senevet et Andarelli, 1956 ; Gaud, 1952, 1953, 1957 ; Gaud et al., 1949; Senevet et al., 1949 ; Senevet et Andarelli, 1954, 1956, 1959; Bailly Choumara, 1965, 1967 ). Par la suite, ces recherches ont été complétées par des investigations dans différentes localités du Maroc (Benjaber, 1985; Metge, 1986; Himmi, 1991, 2007; Trari, 1991; Trari et al., 2003 ; Bouallam, 1992 ; Louah, 1995 ; Chlaida et Elmardi, 1995 ; Handaq, 1998 ; Handaq et Boumezzough, 1999, 2002; El Ouali Lalami et al., 2010 ; 
Amraoui et al., 2012). Ces travaux de recherche bien qu'ils constituent une base importante pour la connaissance des Moustiques du Maroc, ils ne présentent que des contributions locales que nous tenons à compléter par la présente étude, réalisée dans le périmètre urbain et péri-urbain de la ville de Meknès (centre nord du Maroc).

Si d'importants travaux sur l'hydrobiologie des habitats du peuplement culicidien ont été réalisés dans le Nord du Maroc (Louah, 1995 ; El Joubari et al., 2014), la bioécologie des culicidae que l'on pensait bien connaitre reste à préciser, particulièrement celles des régions urbaines et péri-urbaines de la région de Meknes, caractérisées par des activités anthropiques variées (activités agricoles, industries croissantes, urbanisation accrue et aménagement des espaces). Ces régions offrent, en effet, une grande hétérogénéité d'unités paysagères, auxquelles s'intègrent des biotopes d'eaux usées industrielles et ménagères et des eaux stagnantes, d'origine pluviale, constituant des gîtes larvaires souvent temporaires. Ces biotopes, soumis à des conditions drastiques, méritent que l'on étudie l'écologie et le fonctionnement de leurs gîtes abritant les espèces les plus agressives. Le statut taxonomique des espèces et la typologie des gîtes fourniront les éléments indispensables à la mise au point d'éventuelles méthodes de lutte, dirigées contre les moustiques de cette région.

\section{Présentation de la région d'étude}

La région de Meknès appartient au plateau de Saiss, faisant partie du sillon sud-rifain s'étendant sur plus de $100 \mathrm{~km}$ d'Ouest en Est (Fig. 1). Limité au Nord par les rides sud-rifaines (Monts de Zerhoune) et au Sud par les reliefs du Moyen Atlas, ce bassin est bordé du côté Ouest par la vallée de 1'Oued Beht et à l'Est par l'Oued Sebou. La ville de Meknès est située entre les latitudes $33.8441859^{\circ} \mathrm{N}$ et $33.9151584^{\circ} \mathrm{N}$ et les Longitudes $5.4897308^{\circ} \mathrm{E}$ et $5.603714^{\circ} \mathrm{E}$ et à une altitude moyenne d'environ $550 \mathrm{~m}$, sur le plateau dit «Plateau de Meknès», topographiquement plus élevé que la partie Est du même bassin communément appelée « Saiss de Fès ».

La ville impériale de Meknès se classe parmi les six grandes villes du Royaume, avec une population d'environ 800.000 habitants. C'est une ville qui occupe une position stratégique, en représentant un point de passage obligé entre les plaines atlantiques et les hauts plateaux de l'oriental d'une part, et entre le Moyen Atlas, le Maroc central et le Maroc septentrional d'autre part. La région de Meknès offre des potentialités agricoles importantes, les terres fertiles du Saiss et la présence de nappes d'eau phréatique superficielles et profondes constituent des atouts pour toute la région.

Le climat de la région de Meknès est de type méditerranéen tempéré à semi-aride, avec un indice d'aridité de 19,23 $\mathrm{mm} /{ }^{\circ} \mathrm{C}$. Il est caractérisé par 
un régime pluviométrique qui comporte un maximum en hiver et un minimum en été. La période estivale à sécheresse accentuée commence au mois de mai et s'achève au mois de septembre (Fig. 2). La période hivernale, souvent pluvieuse, dure d'octobre à avril avec des maximas en décembre, janvier et février. Les précipitations annuelles maximales connaissent des fluctuations importantes d'une année à l'autre, avec une moyenne de 837,3 $\mathrm{mm}$ en 1995-1996 et seulement 234,9 mm en 1994-1995. D'une manière générale, on note que la moyenne annuelle des précipitations dans la station de Meknès se situe entre 500 et $600 \mathrm{~mm}$.

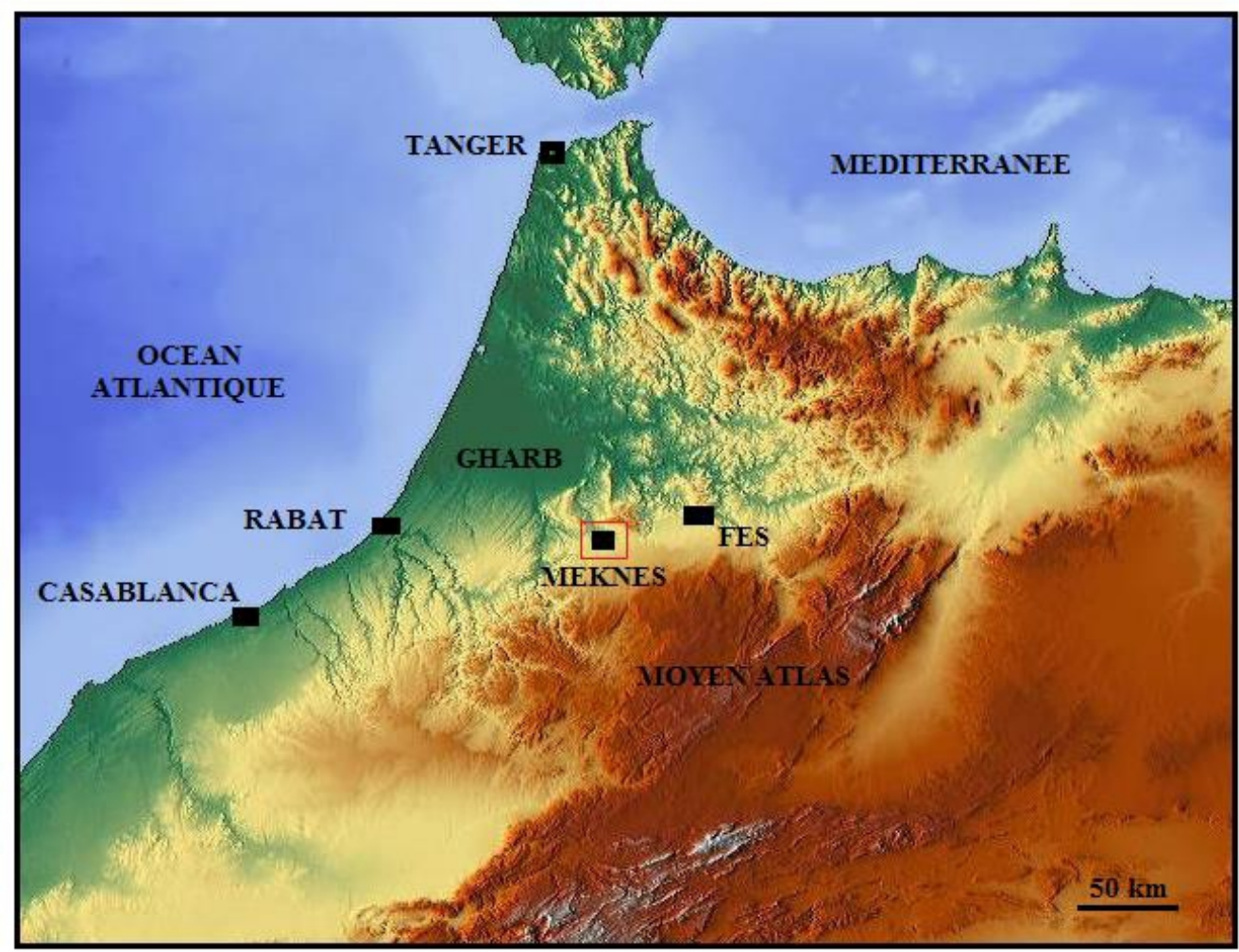

Figure 1 : Localisation de la région étudiée (région de Meknès) dans la partie Nord du Maroc (Extrait MNT du Maroc, site web TWCC.fr)

Lors de fortes pluies dans les zones urbanisées, faute d'infiltrations des eaux par les sols imperméables, les eaux ruissellent, saturent les réseaux d'évacuation et entrainent une montée d'eaux par les égouts ce qui a pour conséquences la création de gîtes potentiels à moustiques (Merja, daya, mare, etc.). 


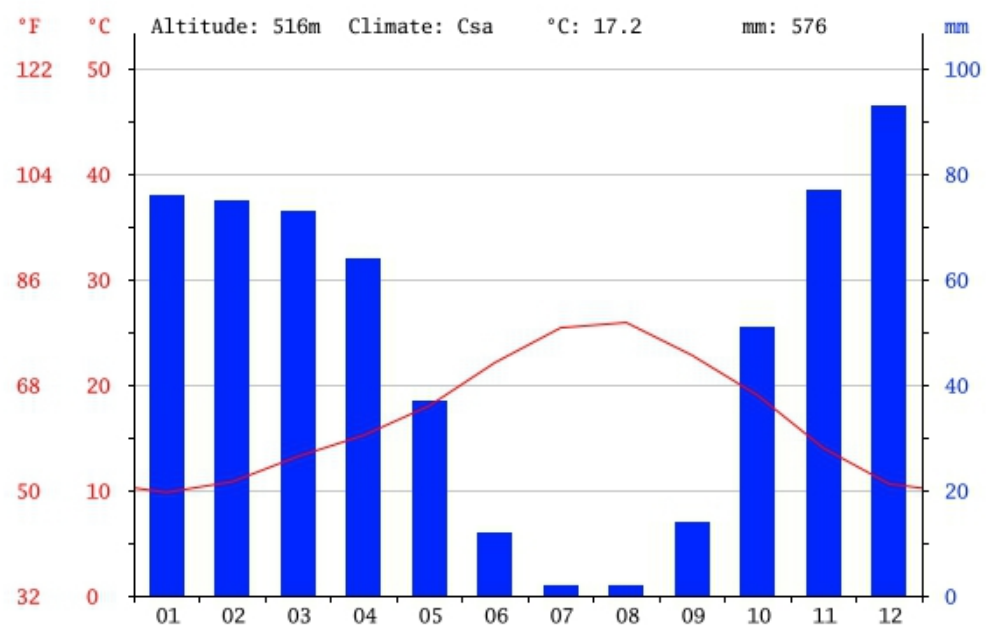

Figure 2 : Diagramme climatique de la région de Meknès 2013-2014 (source site web)

Les températures maximales sont enregistrées aux mois de juillet et août, la température moyenne est de $26,63^{\circ} \mathrm{C}$, par contre le mois le moins chaud est celui de janvier avec des températures de l'ordre de $10,06^{\circ} \mathrm{C}$ (Fig. $3)$.

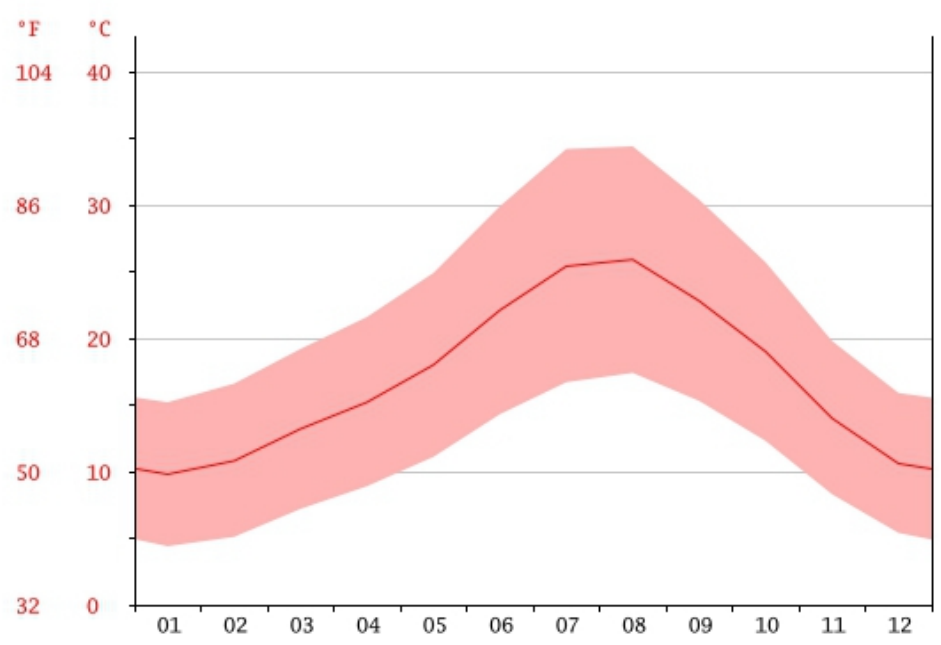

Figure 3 : Courbe des températures de la région de Meknès durant la période d'étude

La différence de précipitations entre le mois le plus humide est de 91 $\mathrm{mm}$. Une différence de $16,1^{\circ} \mathrm{C}$ existe entre la température la plus basse et la température la plus élevée sur toute l'année. 


\section{Description du périmètre urbain de la ville de Meknès irrigué par les eaux usées}

Dans la région de Meknès, une grande partie des espaces verts urbains et périurbains sont irrigués par les eaux usées dont le débit a considérablement augmenté ces dernières années suite à l'ampleur de l'urbanisation. Ces eaux usées sont tantôt mélangées aux eaux claires et tantôt utilisées à l'état brut (Kharrouch et Chahlaoui, 2009).

Ces habitats agricoles longeant les trois vallées de Bou Ishak, Boufekrane et Ouislane constituent un atout de la ville de Meknès (Fig. 4), cependant ils sont à l'origine de nombreuses nuisances à la santé publique et ce par la contamination des sols, des nappes, des eaux superficielles et par la prolifération des espèces culicidiennes, à l'origine des nuisances causées à la population urbaine, surtout pendant la saison estivale (Handaq et Blenzar, 2013).

L'utilisation des eaux usées ou mélangées souvent non traitées dans l'irrigation des différentes cultures dans le périmètre urbain de Meknès est une pratique ancienne et courante. Toutes les communes rurales et les municipalités de Meknès sont concernées par cette pratique. Il s'agit des municipalités de Ouislane, Hamria et Al Ismailia et des deux communes de Dkhissa et Toulal.

Les surfaces cultivées se concentrent surtout en aval de la ville de Meknès et les cultures annuelles occupent les grandes surfaces irriguées. Les surfaces cultivées en blé représentent environ 50\% de la superficie, ensuite les légumineuses, les fourrages et les oléagineuses. De même, toutes sortes de cultures maraîchères sont également pratiquées, il s'agit essentiellement de la pomme de terre et l'oignon. L'olivier est peu répandu, il est planté surtout dans quelques terrains accidentés au niveau des vallées encaissées, ainsi qu'en bordure des routes et des parcelles de cultures. 


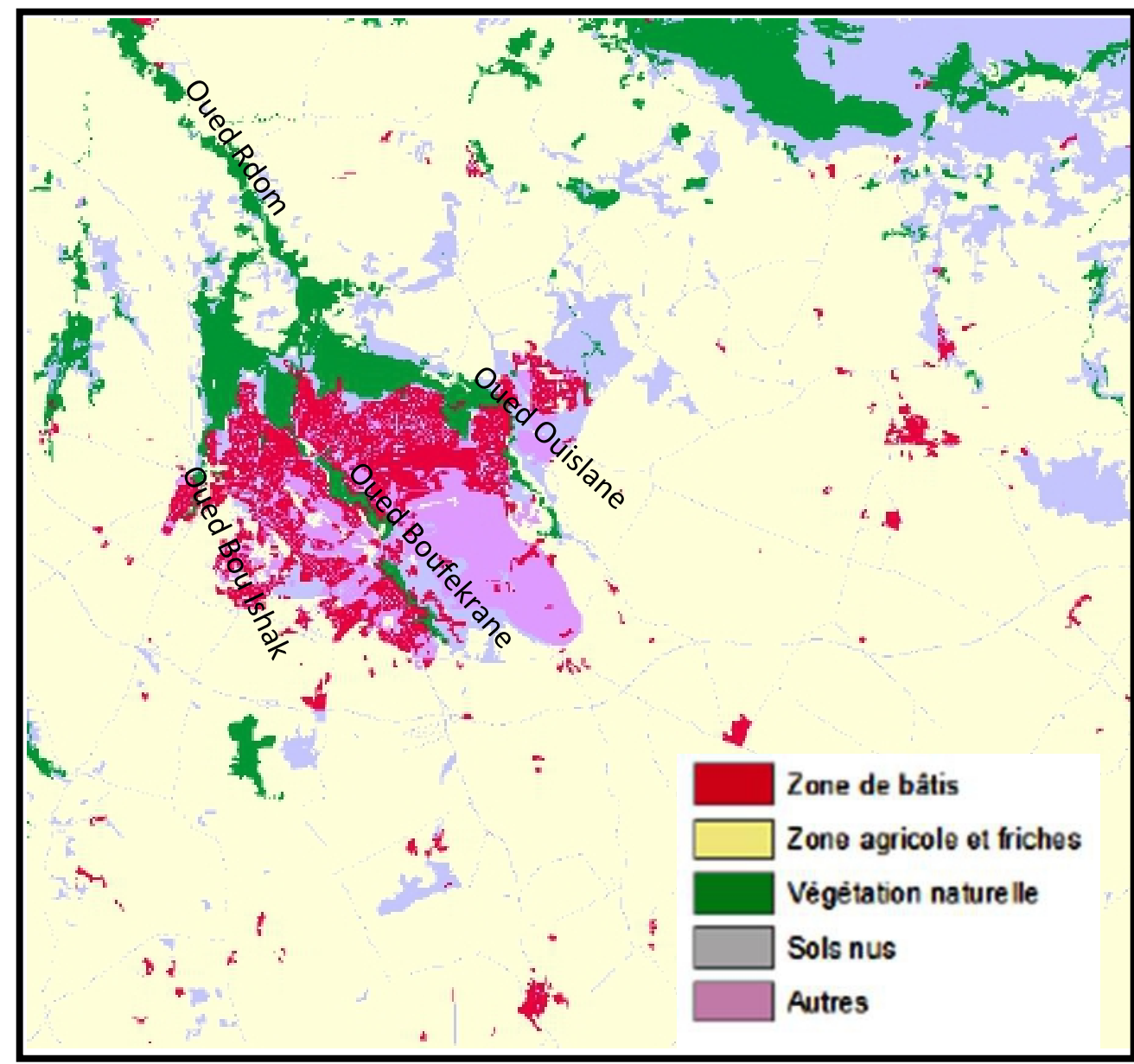

Figure 4 : Espaces verts agricoles et occupation des sols dans la région de Meknès (d'après Azodjilande, 2013, modifiée)

Le long de la vallée de Boufekrane, coulant au milieu de la ville et traversant Meknès depuis ses banlieues sud jusqu'à ses quartiers nord, les terrains irrigués sont situés surtout près de Sidi Bouzekri, Jbabra, Beni Mhamed, Dar El Beida et Sahrij El Ma. Ces espaces constituent des habitats souvent favorables au développement de larves de Culicides, tout particulièrement au niveau des petites stagnations entre les parcelles cultivées. Les terrains irrigués le long de l'oued Ouislane sont situés entre le pont de la route Meknès-Fès et la localité de Ouarzigha, la rive droite est irriguée par des eaux mixtes, par contre la rive gauche est irriguée par les eaux usées brutes. Enfin, le long de Bou Ishak, les terres irriguées sont situées du côté de Sidi Baba, mais aussi près de Toulal. Il s'agit surtout le long de ce vallon, d'une réutilisation des eaux usées brutes. 


\section{Typologie des gîtes larvaires prospectés}

Nos prospections dans la région de Meknès, nous ont permis de repérer dix gîtes larvaires répartis le long des principales vallées traversant la ville (Fig. 5). Les gîtes 1 et 2 sont situés à l'amont de l'agglomération de Meknès, les gîtes 7, 8 et 9 à l'val de la ville, par contre les gîtes 3, 4, 5, 6 et 10 sont situés dans le périmètre urbain.

\section{Gîte G1}

Situé sur les berges de l'Oued Boufekrane à l'aval de la ville de Boufekrane, il est caractérisé par des eaux douces claires, avec des plantes en général, de type hydrophytes et des mousses. Le gîte n'est pas touché par les nuisances causées par les activités anthropiques, à part les activités agricoles (Fig. 6 a). La température moyenne de l'eau est de $15^{\circ} \mathrm{C}$; le $\mathrm{Ph}$ est à tendance basique, nou avons enregistré une moyenne de 7,8. Le taux d'oxygène dissous enregistré traduit une bonne oxygénation de ce gîte (25 $\mathrm{mg} / \mathrm{l})$, le tapis végétal est riche en macrophytes du genre Phragmites et Typha.

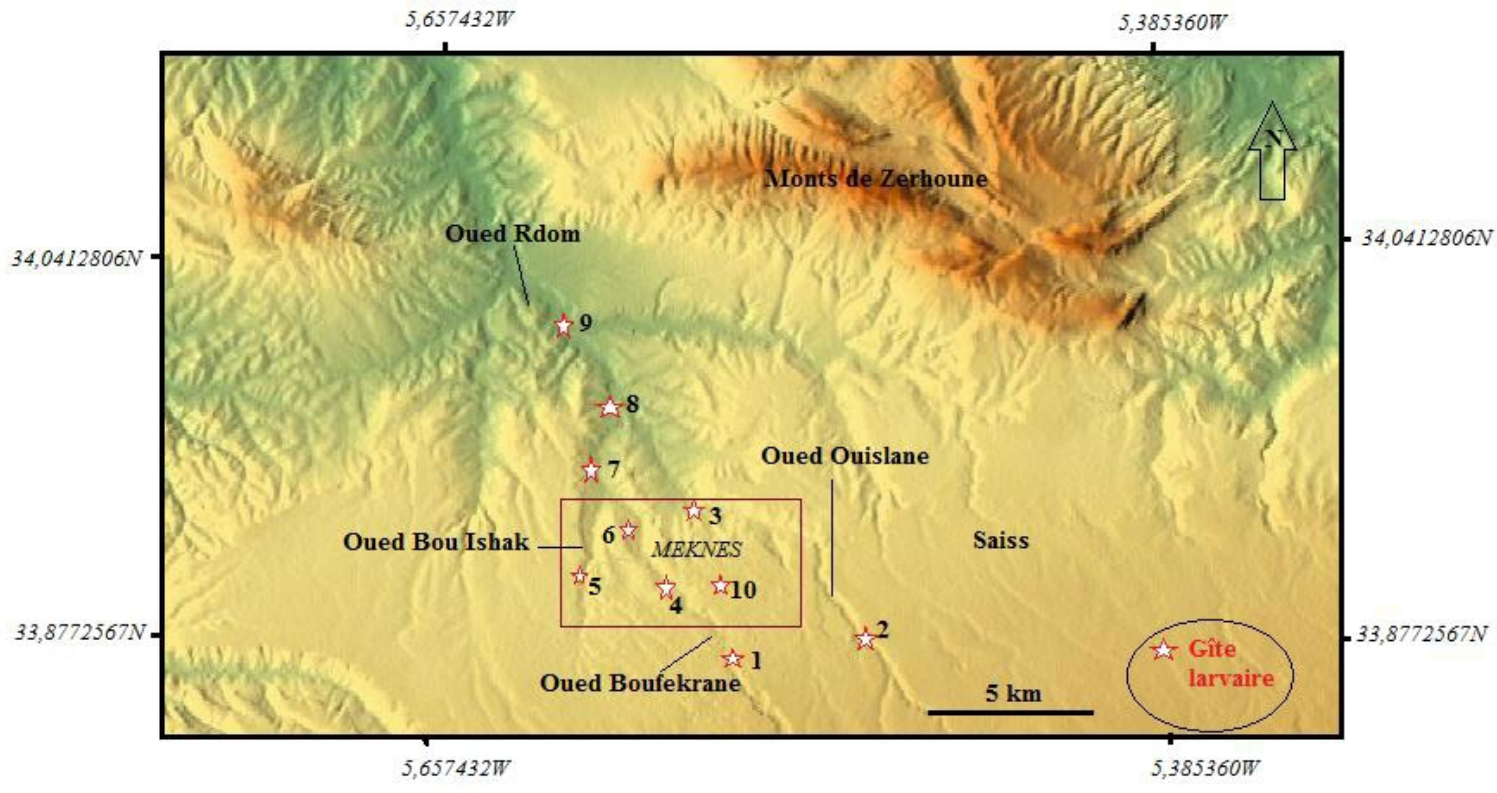

Figure 5 : Répartition des gîtes larvaires étudiés le long des trois vallées traversant la ville de Meknès.

\section{Gîte G2}

Ce gîte est situé sur l'Oued Ouislane à l'aval de la zone industrielle de Sidi Slimane Moul El Kifane. Ce gîte est caractérisé par des cultures saisonnières de maïs pendant la saison estivale (Fig. 6 b, c, d)). Comme c'est le cas dans toute la région de Meknès, les activités agricoles sont pratiquées 
sur l'ensemble du bassin de Saiss (vastes domaines agricoles) ou directement dans les petites parcelles aux bordures des Oueds (activités agricoles saisonnières). La température moyenne de 1 'eau est de $25^{\circ} \mathrm{C}$; le $\mathrm{Ph}$ est à légèrement acide, une moyenne de 6,8 a été enregistrée. Le taux d'oxygène dissous enregistré est de $9 \mathrm{mg} / \mathrm{l}$ ), le tapis végétal est représenté par des algues filamenteuses.

\section{Gîte G3}

Ce gîte est situé le long de l'Oued Ouislane au niveau du pont de Ouislane reliant le quartier El Bassatine et le quartier Ouislane. Ce gîte est caractérisé par des eaux claires mélangées aux eaux usées domestiques. Aux alentours du gîte, prolifèrent des hydrophytes, des mousses et des roseaux.
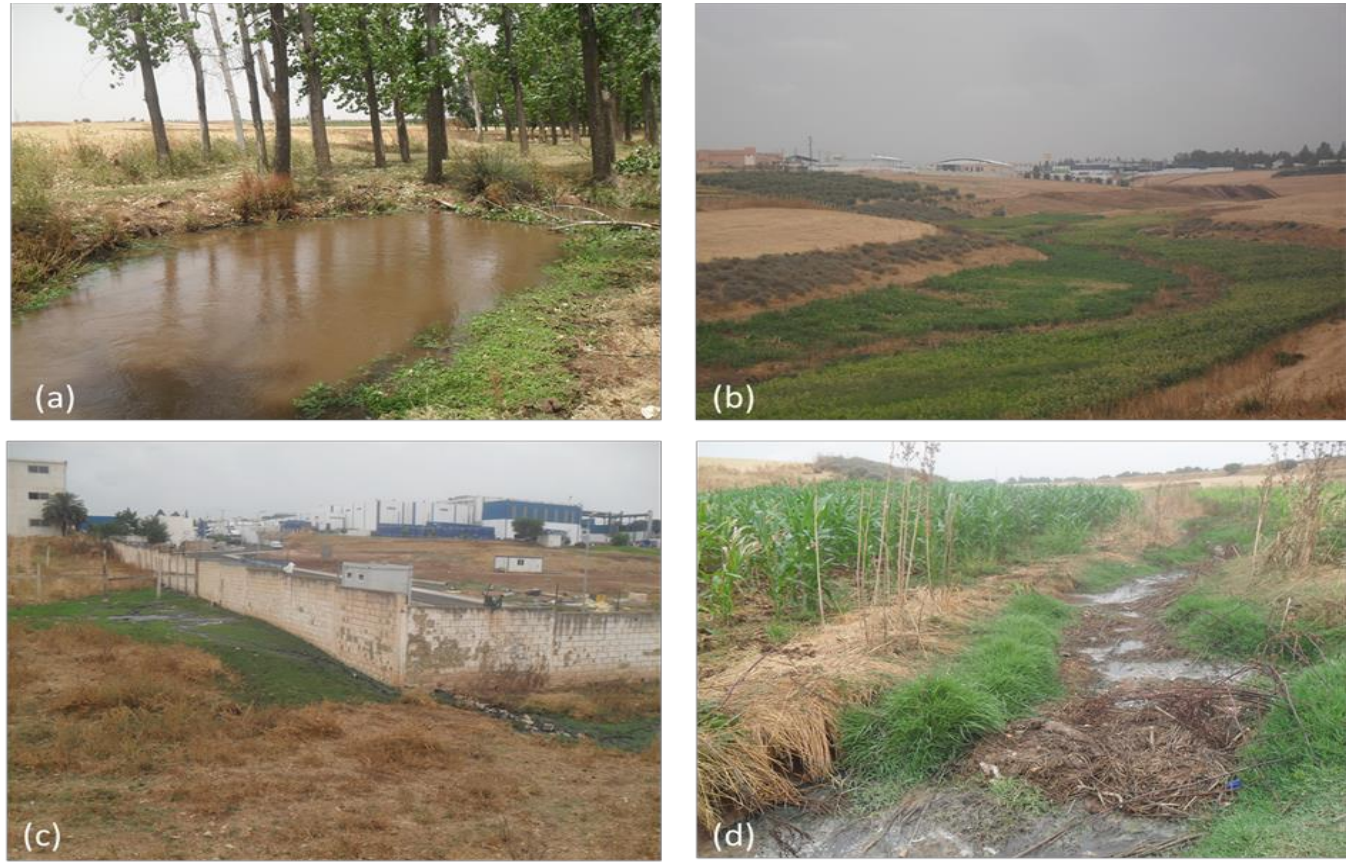

Figure 6 : Différents gîtes larvaires (G1 et G2) prospectés le long de l'Oued Boufekrane (a) et de l'Oued Ouislane près de la zone industrielle de Sidi Slimane MoulKifane (b, $c$ et d).

\section{Gîte G4}

Ce gîte situé le long de l'Oued Boufekrane, à la sortie de l'ancienne Médina de Meknès, près du quartier Fakharine, est caractérisé par des eaux usées domestiques. La teprérature moyenne de l'eau est de $22,5^{\circ} \mathrm{C}$. La teneur moyenne en oxygène dissous est de $12,6 \mathrm{mg} / 1$. Le couvert végétal y est très diversifié, il correspond à cultures maraichères et des hydrophytes le long de lit de l'Oued . 


\section{Gîtes G5 et G6}

Ces deux gîtes sont situés respectivement sur les rives de l'Oued Bou Ishaq et Boufekrane, à l'aval de l'agglomération du grand Meknès. Ils sont caractérisés par un mélange d'eaux claires et d'eaux usées domestiques. La température moyenne de l'eau est de $25^{\circ} \mathrm{C}$; le $\mathrm{Ph}$ est légèrement acide, une moyenne de 6,8 a été enregistrée. Le taux d'oxygène dissous enregistré est de $10,5 \mathrm{mg} / \mathrm{l})$, le tapis végétal coorespond à des parcelles agricoles à cultures variées et alternées, irriguées par ces eaux.

\section{Gîtes G7 et G8}

Le gîte G7 est situé sur l'Oued Bou Ishak, juste à l'amont de la station de traitement des eaux usées, avant le point de confluence des deux oueds Bou Ishak et Boufekrane. Ce gîte G7 reste naturel et présente un caractère temporaire en relation avec les activités agricoles le long de cette vallée. Par contre, le gîte G8 est situé au point de confluence des trois vallées Bou Ishak, Boufekrane et Ouislane, marquant le début de l'Oued Rdom.

\section{Gîte G9}

Le gîte G9 à création artificielle, correspond à des bassins en caoutchouc noir, aménagés au niveau de la station des services Petrom, située sur la route Meknès-Sidi Kacem $\left(33.9757784^{\circ} \mathrm{N}, 5.5827686^{\circ} \mathrm{E}\right.$, alt : $286 \mathrm{~m})$.

Il s'agit de bassins rectangulaires métriques, remplis soit artificiellement, soit par les eaux de pluie, pour irriguer des plantes ornementales implantées en dehors de la station (Fig. 7). 

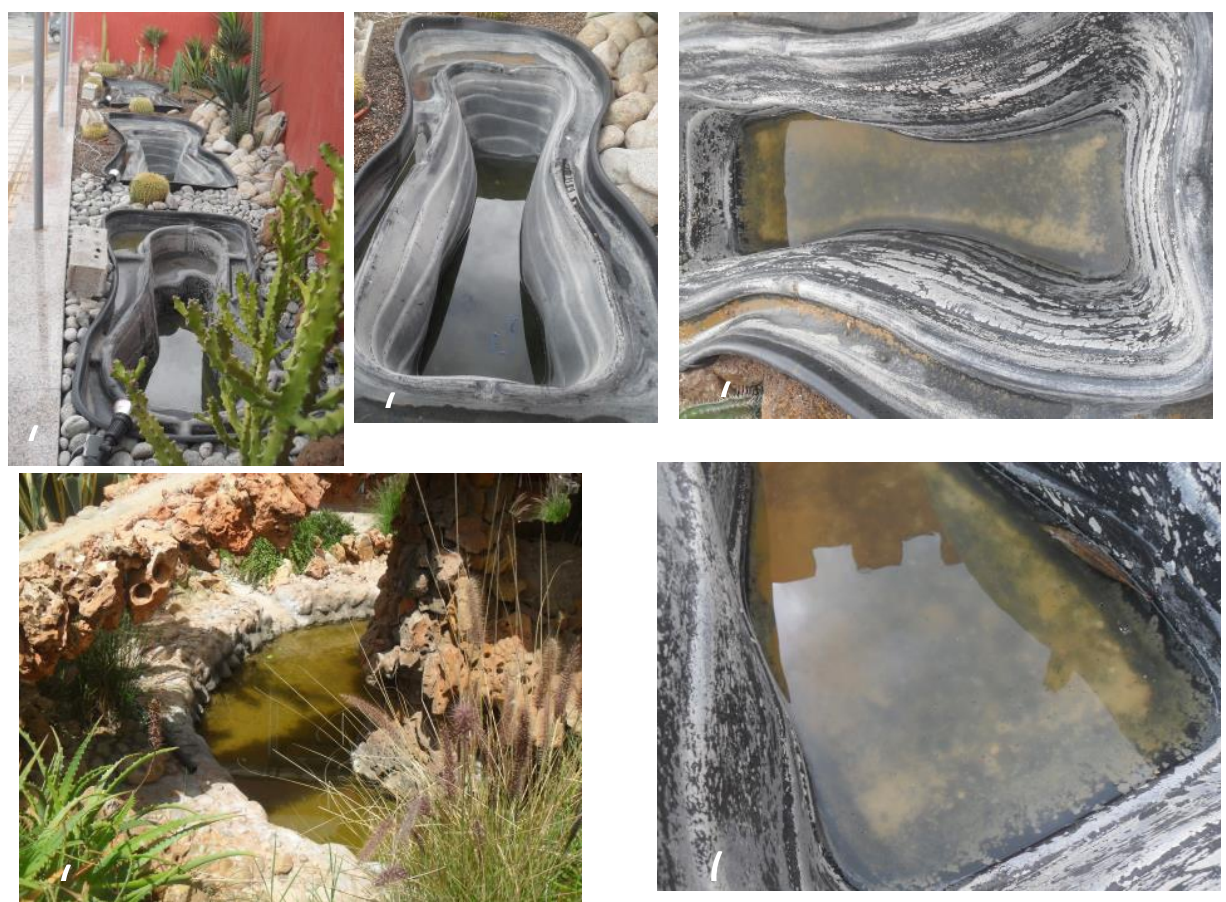

Figure 7 : Types de gîtes larvaires créés suite au réaménagement de la station des services de l'Oued Rdom (G9) (a) : panorama des trois bacs aménagés pour l'irrigation des plantes ornementales de la station des services, (b) : bac rectangulaire constituant l'un des gîtes, (c) :

bac à moitié rempli par les eaux de pluies, (d) : gîte situé à l'intérieur de la station des services correspondant à une fontaine, (e) : détail du gîte à eau de pluie montrant la présence de larves de moustiques).

\section{Gîte G10}

C'est un gîte particulier car il est situé en plein centre-ville de Meknès, le long du Boulevard principal d'Ibn Sina $\left(33.8975633^{\circ} \mathrm{N}\right.$, $5.5319595^{\circ} \mathrm{E}$, alt : $539 \mathrm{~m}$ ).

Ce gîte a été découvert par hasard. Il s'agit des différentes fontaines publiques aménagées le long du Boulevard Ibn Sina et dans le jardin public en face de la gare ONCF (Gare de Meknès). Il correspond à trois petits bassins rectangulaires, encadrés par un espace gazonné restreint, et deux rangées de palmiers (Fig. 8). Le second gîte correspond à une fontaine circulaire, elle aussi entourée par un espace gazonné et des palmiers de petite taille (photo 3). 
Le suivi de ce gîte permanent, pendant plus d'une année, depuis avril 2015 jusqu'au mois d'octobre 2016, nous a permis d'enregistrer une température moyenne de $22^{\circ} \mathrm{C}$. La teneur en oxygène dissous est de $7 \mathrm{mg} / 1$. Celle des chlorures est de $1.5 \mathrm{mg} / 1$. Nous avons aussi enregistré une teneur en matière organique de $17 \mathrm{mg} / \mathrm{l}$.
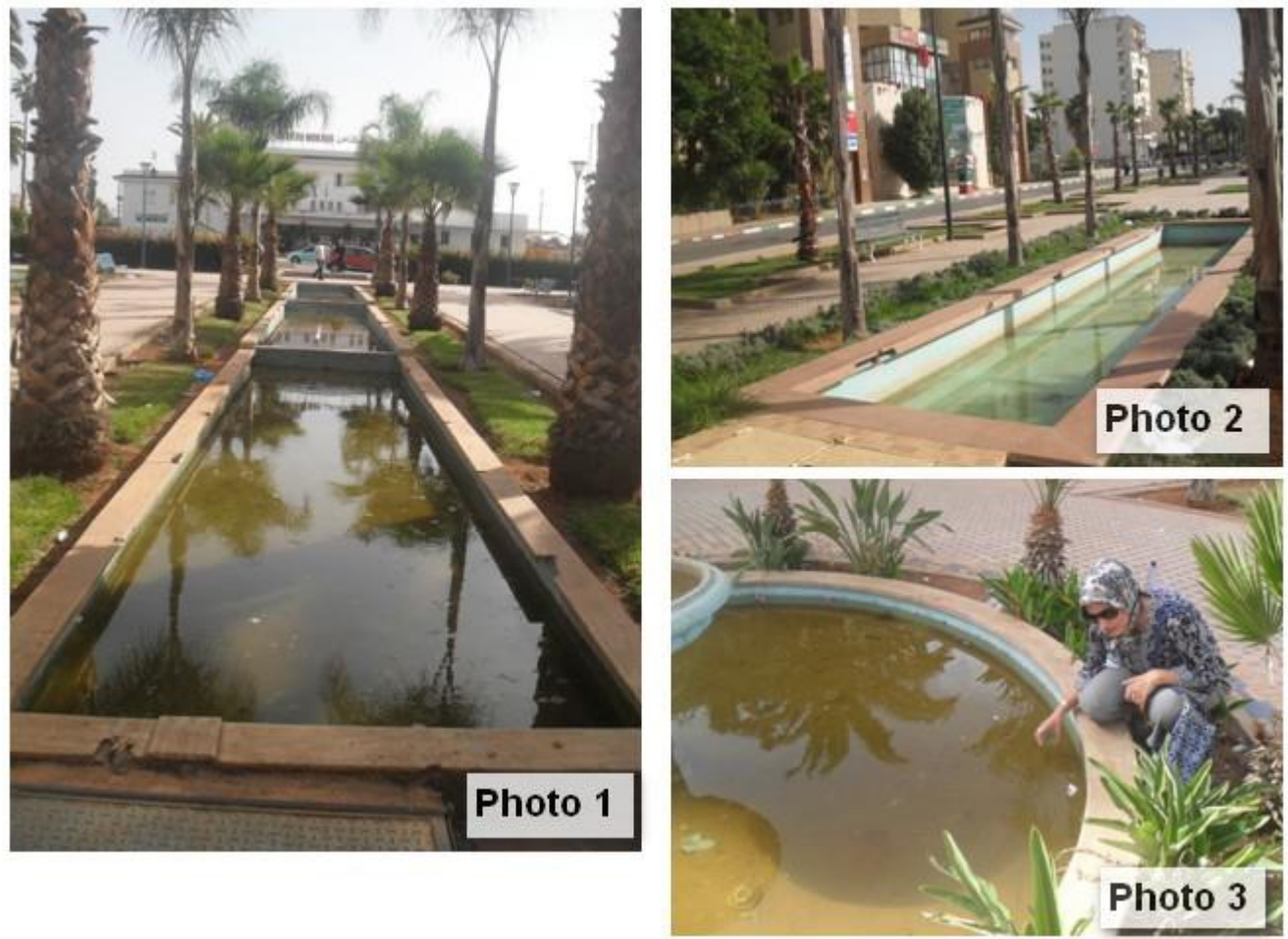

Figure 8 : Clichés des principaux gîtes prospectés dans le jardin public en face de la gare de Meknès (G10).

\section{Inventaire des espèces culicidiennes}

Le suivi physico-chimique des gîtes larvaires, rencontrés dans le périmètre étudié, a permis de mettre en évidence les principaux éléments qui conditionnent la répartition des espèces culicidiennes. Celle-ci dépend en grande partie de la situation des gîtes soit en amont des rejets des eaux usées (banlieue sud de la ville de Meknès) soit à l'aval de ces rejets. Ainsi dans les gîtes G1 et G2 (Fig. 9), nous avons recensé 4 genres Anopheles, Culex, Aedes et Culiseta. Les espèces recensées sont: Anopheles labranchiae, Anopheles claviger, Anopheles algeriensis, Culex deserticola, Culex hortensis, Culex theileri, Culex univittatus, Aedes vexans, Aedes rusticus, Aedes caspius et enfin Culiseta longiareolata.

De même, dans les gîtes G3 à G8, situés dans le périmètre urbain recevant les eaux usées domestiques et industrielles, a faible teneurs en oxygène dissous et très riches en matière organique, nous avons recensé 
surtout la prédominance de Culex pipiens lors de toutes nos pêches larvaires avec parfois la présence d'Aedes caspius (Fig. 9).

Enfin dans les gîtes 9 et 10 , correspondant à des gîtes liés à des travaux d'aménagement urbain (fontaines de jardins publics ou privés), nous avons noté la prédominance de l'espèce Culex pipiens (67\%) avec parfois la présence de Culiseta longiareolata (25\%) et d'Anopheles labranchae (8\%).

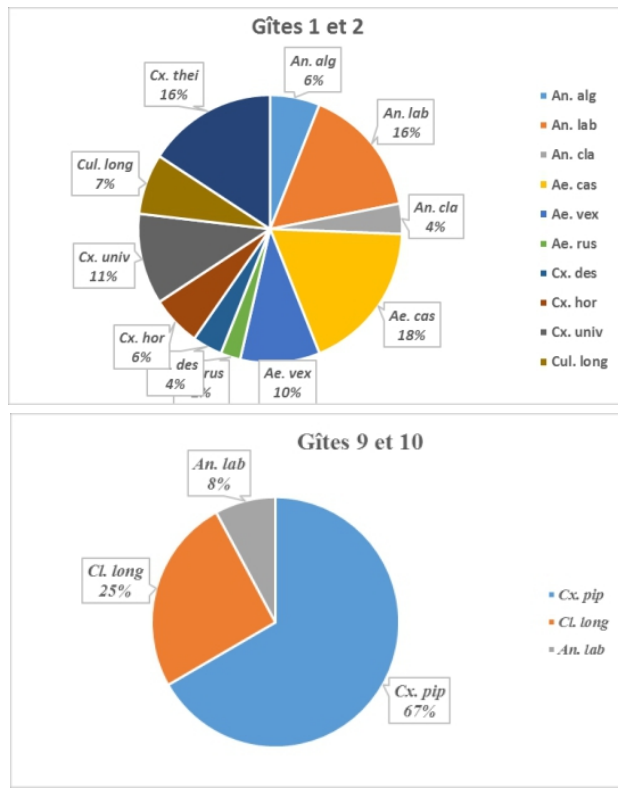

Figure 9 : Aspects quantitatifs et qualitatifs des espèces de Culicidae recensées dans les gîtes urbains et péri-urbains de la ville de Meknès (pêches larvaires réalisées au mois de mai 2015).

\section{Discussion et conclusions}

L'inventaire des espèces culicidiennes recensées dans les différents gîtes larvaires dans le périmètre urbain et péri-urbain de la ville de Meknès montre que la nature du gîte influence grandement la distribution des espèces des culicides. La nature du gîte larvaire favorise la présence de certaines espèces et non d'autres selon que ce gîte est à eau stagnante ou courante, dépourvu ou riche en végétation, pollué ou non. Ainsi, les gîtes G1 et G2 situés dans la banlieue sud de Meknès, en amont des rejets des eaux usées domestiques et industrielles, à couvert végétal composé essentiellement d'herbes (Typha angustifolia, Lemna gibba), de roseaux (Juncus biloba) et d'algues filamenteuses montrent une richesse spécifique importante avec la présence de onze espèces réparties en quatre genres. Par contre dans les gîtes G3 à G8, caractérisés par des eaux polluées, à forte teneur en matière organique, à $\mathrm{pH}$ moyen de 8,2 et taux de chlorures de, nous avons noté la présence uniquement de deux espèces appartenant à deux genres Culex et 
Aedes. La réduction de la richesse spécifique constatée au niveau des gîtes G3 à G8 est en relation avec la dégradation de la qualité des eaux, liée principalement aux rejets des eaux usées domestiques et industrielles. Seule l'espèce Culex pipiens prolifère dans ce type de gîtes (Trari, 1991, Himmi, 1991, 2007 ; Bouallam, 1992 ; Handaq, 1998 ; Handaq et Boumezzough, 1999 ; El Ouali Lalami et al., 2009), espèce constituant 90\% des pêches larvaires. Ce qui confirme le caractère urbain et ubiquiste de l'espèce et toute la nuisance qu'elle cause pour l'agglomération urbaine de la ville de Meknès.

Les prospections des différents gîtes larvaires de Culicidés dans le périmètre urbain et péri-urbain de la ville de Meknès, pendant 3 années successives, à des intervalles réguliers, ont révélé l'existence dans la région de onze espèces, appartenant à quatre genres de deux sous-familles : Anophelinae et Culicinae. Pour ne citer que ces espèces, Culex pipiens est largement abondant dans le périmètre urbain, par contre Aedes caspius abonde dans les gîtes situés en aval de la ville de Meknès, le long de l'Oued Rdom. Enfin, l'espèce Anopheles labranchiae est principalement rencontrée temporairement en amont, le long de l'Oued Boufekrane, là où les eaux sont claires. Les gîtes larvaires prospectés sont soit liés ou non à la pratique d'irrigation par les eaux usées domestiques et industrielles, soit liés aux aménagements des espaces de loisirs ou d'embellissement de la ville de Meknès. Les gîtes prospectés dans le jardin public de la gare ONCF de Meknès, en constituent de bons exemples. Ces gîtes ne nous ont livré que rarement une riche faune culicidienne probablement suite aux traitements par insecticides de ces espaces publics.

\section{References:}

1. Alaoui Slimani, N., Jouid, N., Benhoussa, A. \& Hajji, K. (1999). Typologie des habitats d'Anopheles dans une zone urbaine (Diptera, Culicidae). L'Entomologiste 55 (5) :181-190.

2. Amraoui, F., Krida, G., Bouattour, A. \& Rhaiem, A. (2012). Culex pipiens, an experimental efficient vector of west Nile and Rift Valley fever viruses in the Maghreb region. journal plos.org

3. Azodjilande, J.F. (2013). Cartographie diachronique de l'occupation du sol en zone périurbaine et analyse spatiale des changements d'usage du sol à Meknès (Maroc). Mém. de Master, Univ. Jean Monnet, Saint Etienne, Fr., 82p.

4. Bailly Choumara, H. (1965). Présence de Mansonia pulchripolis (Diptera-Culicidae). Bull.Soc.Sc.Nat.Phys. Maroc,1 er et 2eme trimestre., pp.39-42.

5. Bailly Choumara, H. (1967). Rapport d'une mission entomologique effectuée dans la vallée de la Moulouya. Lab.Entomol., I.S.C., Maroc, 11p. 
6. Bailly Choumara, H. (1973 a). Etude comparative de différentes techniques de récoltes de moustiques adultes (Diptera-Culicidae) faites au Maroc en zone rurale. Bull. Soc. Nat. Phys. Maroc,1er et 2ème trimestre, 53, pp.135-188.

7. Bailly Choumara, H. (1973 b). Etude préliminaire d'une récolte d'Anopheles labranchae par piège C.D.C réalisée dans la région de Larache, Maroc. Bull. OMS, 49, pp. 49-55.

8. Benjaber, M. (1985). Contribution à l'étude dendrolimnique de la subéraie en pays Zaer (Maroc). Aspect systématique. DEA Univ. Paul Sabatier, Toulouse, 113p.

9. Bouallam, S. (1992). Le paludisme et les moustiques dans la région de Marrakech. Ecologie et cycles biologiques des espèces culicidiennes. Thèse de 3eme cycle, Université Cadi Ayyad, Faculté des Sciences de Marrakech.

10. Chlaida, A. \& Elmdari N. (1995). Dynamique des populations et cartographie écologique des gîtes larvaires de quelques espèces des Diptères Culicidés au niveau de la retenue du barrage EL Massira. Actes.AML, Fes, 20et 21 Déc.

11. El Joubari M., Louah A. \& Himmi, O. (2014). Les moustiques (Diptera,Culicidae) des marais de Smir (nord-ouest du Maroc) : inventaire et biotypologie. Bull.Soc.Path.Exot, 107, pp.48-59.

12. El Ouali Lalami, A., Hindi, T., Azzouzi, A., El Ghadraoui, L., Maniar, S., Faraj, C., Adlaoui, E., Ameur, I. \& Ibnsouda Koraichi, S. (2009). Inventaire et répartition saisonnière des Culicidae dans le centre du Maroc. Entomologie faunistique, 62 (4), pp. 131-138.

13. El Ouali Lalami, A., Hindi, A. \& Azzouzi A. (2010). Inventaire et répartition saisonnière des Culicidae dans le centre du Maroc. Entomologie faunistique- Faunistic Entomology 62 (4):131-138.

14. Faraj, C., Elkohli, M. \& Lyagoubi, M. (2006). Cycle gonotrophique de Culex pipiens (Diptera: Culicidae), vecteur potentiel du virus West Nile, au Maroc: estimation de la durée en laboratoire. Bull. Soc. Path. Exot. 99 (2):119-12.

15. Faraj, C., Adlaoui, E. \& Brengues, C. (2008). Résistance d'Anopheles labranchiae au DDT au Maroc: identification des mécanismes et choix d'un insecticide de remplacement. East Mediterr. Health J. 14 (4) : 776-783.

16. Gaud, J. (1952). Données sur la biocénose culicidienne de quelques gîtes du Gharb en 1952. Bull.Inst.Hyg. Maroc, 12, (1-2) : pp.55-72.

17. Gaud, J. (1953). Notes biogéographiques sur les Culicidae du Maroc. Arch. Inst. Past. Maroc 4 (7) : 443-490. 
18. Gaud, J. (1957). Présence au Maroc de Theobaldia Subochrea Edward (Diptera : Culicidae). Arch. Inst. Past. Maroc, 5 (6) : 268269.

19. Gaud, J., Faure, F. \& Maurice, A. (1949). Biogéographie des espèces anophéliennes au Maroc. Bull. Inst. d'Hyg. du Maroc, 9, pp.145-163.

20. Guy, Y. (1963). Bilan épidémiologique du paludisme au Maroc (données recueillies en 1960,1961 et 1962). Ann. Parasit. hum. comp.,38, (5), pp. 823-857.

21. Guy, Y. \& Holsteun, M. (1968). Données récentes sur les Anopheles du Maghreb. Arch.Inst.Past.Algérie, 45, pp. 51-61.

22. Guy, Y., Salieres, A. \& Boesiger, E. (1976). Contribution à l'étude du complexe maculipennis (Diptera - Culicidae - Anophelinae). Mise au point en 1975. Annales de Biologie, 15, pp. 5-6.

23. Handaq, N. (1998). Les Moustiques du Maroc: Ecologie et biogéographie des peuplements culicidiens dans les régions montagneuses, semi-arides et arides du Maroc occidental. Essai de biotypologie des gîtes larvaires, étude comparative de la dynamique des populations marocaines et tunisiennes. Thèse de 3eme cycle, Univ. Cadi Ayyad, Marrakech, Faculté des Sciences Semlalia, 193p.

24. Handaq, N. \& Boumezzough, A. (1999). Aedes caspius dans le sudouest marocain : impact de l'aridité sur la dynamique des populations préimaginales (Diptera, Culicidae). Bull. Soc. Ent. Fr., Vol. 104, n², pp. 183-191.

25. Handaq, N. \& Boumezzough, A. (2002). Confirmation de la présence au Maroc d'Aedes aegypti Linné, en zone continentale dans la région de Marrakech (Dipt., Culicidae). Bull. Soc. Ent. Fr., Vol. Tome 107, 2002, p. 200.

26. Handaq, N. \& Blenzar, A. (2013). Les Aedes du Maroc (DipteraCulicidae): Etude taxonomique et biogéographique de 5 espèces d'Aedes. SciLib, Vol. 6, $\mathrm{N}^{\circ}$ 140602, ScienceLib. Editions Mersenne, pp. 1-15.

27. Himmi, O. (1991). Culicidae (Diptera) du Maroc : clé de détermination actualisée et étude de la dynamique et des cycles biologiques de quelques populations de la région de Rabat-Kénitra. Thèse de 3ème cycle, Fac. Sciences de Rabat, 185 p.

28. Himmi, O. (2007). Les culicidés (Insectes, diptères) du Maroc: Systématique, écologie et études épidémiologiques pilotes. Thèse doct d'Etat, Université Mohamed V, Faculté des Sciences de Rabat.

29. Karrouch, L. \& Chahlaoui, A. (2009) - Bio-évaluation de la qualité des eaux de l'oued Boufekrane (Meknès, Maroc). Biomatec /Volume3/n 6/ pp. 6-17. 
30. Louah, A. (1995). Ecologie des Culicidae (Diptera) et état du paludisme dans la péninsule de Tanger. Thèse d'Etat ès-sciences, Faculté des Sciences de Tétouan, Maroc.

31. Messerlin, A. (1938). L'Aedes mariae au Maroc occidental. Bull. Soc. Path. Exot., 31, (2) : 110-115.

32. Metge, G. (1986). Étude des écosystèmes hydromorphes (Daya et Merja) de la Meseta occidentale marocaine: typologie et synthèse cartographique à objectif sanitaire appliquée aux populations d'Anopheles labranchiae (Falleroni, 1926), (Diptera, Culicidae, Anophelinae). Thèse doct ès-sciences, Université de droit d'économie et des sciences d'Aix-Marseille, France.

33. Metge, G. (1991). Contribution à l'étude écologique d'Anopheles labranchae au Maroc: activité des imagos et dynamique des stades pré-imaginaux de la région Sidi Bettache. Bull. d'Ecologie, 22 (3-4), pp. 419-426.

34. Rioux, J.A. (1958). Les Culicides du Midi méditerranéen. Encyclopédie Entomologique, P Lechevalier (Ed) 35, pp. 303.

35. Roubaud, E. (1935). Variété nouvelle de l'A. maculipennis au Maroc, Anopheles maculipennis sicaulti. Bull. Soc. Path. Exot., 28, pp. 274278.

36. Seguy, E. (1930). Contribution à l'étude des diptères du Maroc. Mém. Soc. Sc. Nat. Phys. Maroc 24, 206 p.

37. Senevet, G., Gaud, J. \& Millet, A. (1949). Validité de l'espèce Culex mauritanicus Callot, 1940. Arch. Inst. Past. Algérie 27 (1) : 42-47.

38. Senevet, G. \& Andarelli, L. (1954). Le genre Aedes en Afrique du Nord:

39. 1-Les larves. Arch. Inst. Past. Algérie 32 (4) : 310-351.

40. Senevet, G.\& Andarelli, L. (1956). Les Anopheles de l'Afrique du Nord et du bassin méditerranéen. Encyclopédie Entomologique, P. Lechevalier Ed., Paris, XXXIII, 280 p.

41. Senevet, G. \& Andarelli, L. (1959). Les moustiques de l'Afrique et du bassin méditerranéen, II: les genres Culex, Uranotaenia, Theobaldia, Orthopodomyia et Mansonia. P. Lechavalier Ed., Paris Encyclopédie Entomologique, XXVII, 384 p.

42. Trari, B. (1991). Culicidae (Diptera) : Catalogue raisonné des peuplements du Maroc et études typologiques de quelques gîtes du Gharb et de leurs communautés larvaires. Thèse de 3ème cycle, Faculté des Sciences, Université Mohamed V, Rabat.

43. Trari, B., Dakki, M., Himmi, O. \& El Agbani, M.A. (2003). Les moustiques (Diptera Culicidae) du Maroc. Revue bibliographique (1916-2001) et inventaire des espèces. Bull. Soc. Pathol. Exot. 96 (4) : 329-334. 